Bull. Austral. Math. Soc.

$46 \mathrm{~B} 20,46 \mathrm{~B} 03$

VOL. 69 (2004) [451-456]

\title{
THE DAY NORM AND GRUENHAGE COMPACTA
}

\author{
M. Fabian, V. Montesinos and V. ZizleR
}

A close connection between the strict convexity of the Day norm to the concept of the Gruenhage compacta is shown. As a byproduct we give an elementary characterisation of Gul'ko compacta in the sigma-product of lines and a more elementary proof of Mercourakis' renorming result for Vašák spaces.

This note is a result of our effort to classify those Banach spaces with dual ball Corson (in its weak ${ }^{*}$ topology) that would admit a Gâteaux smooth renorming.

Given a non-empty set $S$, let $\Sigma(S):=\left\{x \in[-1,1]^{S}\right.$; support of $x$ is countable $\}$. We shall always assume that $\Sigma(S)$ is endowed with its product topology. A compact space $K$ is called a Corson compact if $K$ is homeomorphic to a subset of $\Sigma(S)$.

It was proved in $[1$, p. 425] that a Banach space $X$ with dual ball Corson need not in general admit any equivalent Gâteaux differentiable norm. We find here a sufficient condition for a Gâteaux smooth renorming that uses the strict convexity of the Day norm on the dual space and is closely related to the notion of Gruenhage compacta (see [9], [1, p. 424], [12, Definition 2.1]). As a corollary, we prove a renorming theorem which gives, in particular, a result in [10].

The result in this note is related to the result of Raja that $X^{*}$ admits a dual norm that is weak* locally uniformly rotund if and only if $B_{X}$ - in its weak ${ }^{*}$ topology is a descriptive compact ([11]).

As a byproduct of our efforts, we obtain a characterisation, in the Sokolov's style [13], of Gul'ko compacta lying in the space $\Sigma(S)$ in the spirit of the characterisation of Eberlein compacta given in [8].

Assume that a Banach space $X$ admits a Markushevich basis, that is, a biorthogonal system $\left\{x_{\alpha}, f_{\alpha}\right\}_{\alpha \in \Gamma}$ such that $\overline{\operatorname{span}}\left\{x_{\alpha}\right\}=X$ and $\left\{f_{\alpha}\right\}$ separates points in $X$. Then, it

Received 17th November, 2003

The first author was supported by grants AV 1019003, A 1019301 and GACR 201/01/1198. The second author was supported in part by Project BFM2002-01423 and a grant of the Universidad Politécnica de Valencia. This author thanks the Department of Mathematical and Statistical Sciences of the University of Alberta, Edmonton, Canada, and the Institute of Mathematics of the Czech Academy of Sciences for their support and hospitality. The third author was supported by grant NSERC 7926 .

The authors would like to thank L.E. Garcia Martínez for discussions on the subject of this note and an anonymous referee for some remarks that improved the presentation.

Copyright Clearance Centre, Inc. Serial-fee code: 0004-9727/04 \$A2.00+0.00. 
was shown in [7] and [6] that $X$ admits an equivalent uniformly Gâteaux differentiable norm if and only if the set $\Gamma$ can be split into $\Gamma=U \Gamma_{n}$ such that the formula $\|f\|^{2}$ $:=\|f\|^{2}+\sum D_{n}^{2}(f)$ gives a dual weak uniformly rotund norm on $X^{*}$. Here by $\|f\|$ we mean the original sup norm of $X^{*}$ and $D_{n}(f)$ is the Day norm applied to the coordinate functionals $\left\{f_{\alpha}\right\}_{\alpha \in \Gamma_{n}}$. The norm $\|\cdot\|$ of $X^{*}$ is weak* uniformly rotund if $f_{n}-g_{n} \rightarrow 0$ in the weak* topology whenever $f_{n}, g_{n} \in S_{X^{*}}$ and $\left\|f_{n}+g_{n}\right\| \rightarrow 2$. This property is known to be the dual property to the uniform Gâteaux differentiability of the norm, ([2]). The norm $\|\cdot\|$ on $X$ is uniformly Gâteaux differentiable if $\lim _{t \rightarrow 0} t(\|x+t h\|+\|x-t h\|-2) / t=0$ uniformly for $x \in S_{X}$, the unit sphere of $X$.

This means that if we know that $X$ admits an equivalent Gâteaux differentiable norm, we can use the Day norm on the dual space to construct another uniformly Gâteaux differentiable norm on $X$. therefore the situation is similar to that of uniformly Fréchet differentiable norms (James-Enflo theorem, see for example, [2, Chapter 4] or [4, Chapter 9]).

In [8], the Eberlein compacta lying in $\Sigma(S)$ were classified by using infinite combinatorics.

In [5] and in [6] we characterised several classes of non-separable Banach spaces $X$ by the existence of a total subset (that is, linearly dense) subset $\Gamma \subset X$ which can be split in a certain way. Our method uses only projectional resolutions and techniques in Markushevich bases. In particular, it does not use any combinatorics. Instead, we use the method of projections to characterise Gul'ko compacta in $\Sigma(S)$, in the style of Sokolov [13]. A compact space $K$ is a Gul'ko compact if $(C(K), p)$ is $\mathcal{K}-c d$, where $p$ denotes the topology of the pointwise convergence. A topological subspace $T$ of a compact space $K$ is $\mathcal{K}-c d$ whenever there exist closed sets $K_{n} \subset K, n \in \mathbb{N}$ with the property that for every $t \in T$ and for every $k \in K \backslash T$ there exists $n \in \mathbb{N}$ such that $t \in K_{n}$ and $k \notin K_{n}$. A Banach space $X$ is Vašák (or weakly countably determined), if $(X, w)$ is $\mathcal{K}-c d$. Then, a compact space $K$ is Gul'ko if and only if $(C(K),\|\cdot\|)$ is Vašák, see, for example, [3, Theorem 7.1.8].

We follow the standard notation that can be found, for example, in $[4,3]$. For all concepts and results not explained here we refer to $[4,2,3]$.

Given a subset $\Gamma \subset X$ and a projectional resolution of the identity $\left(P_{\alpha}\right)_{\omega_{0} \leqslant \alpha \leqslant \mu}$ on $X$ (see, for example, [3]), we shall say that they are subordinated (to each other) if $P_{\mathbf{\alpha}}(\gamma) \in\{\gamma, 0\}$ for all $\gamma \in \Gamma$ and $\omega_{0} \leqslant \alpha \leqslant \mu$.

A subset $\Gamma$ of a Banach space $X$ is said to countably support $X^{*}$ if $\#\{\gamma \in \Gamma$ : $\left.\left\langle\gamma, x^{*}\right\rangle \neq 0\right\} \leqslant \aleph_{0}$, for all $x^{*} \in X^{*}$ (here \# denotes the cardinal number of a set). It follows that if a Banach space $X$ has a total subset $\Gamma$ which countably supports $X^{*}$, then there exists a projectional resolution of the identity on $X$ subordinated to $\Gamma$, as $\Phi\left(x^{*}\right):=\left\{\gamma \in \Gamma:\left\langle\gamma, x^{*}\right\rangle \neq 0\right\}$, for all $x^{*} \in X^{*}$, is a projectional generator (see, for example, [3, Definition 6.1.6] and [7]). 
The following result follows by transfinite induction on the density character of $X$. For the concept of separable projectional resolution of the identity see, for example, [3, Definition 6.2.6].

Proposition 1. Let $X$ be a weakly countably determined Banach space. Let $\Gamma \subset X$ be a total subset of $X$ that countably supports $X^{*}$. Then there exists a separable projectional resolution of the identity on $X$ subordinated to $\Gamma$.

A subset $\Gamma \subset X$ of a Banach space $X$ will be called weakly $\sigma$-shrinking if there exists a sequence $\left(\Gamma_{n}\right)_{n=1}^{\infty}$ of subsets of $\Gamma$ such that $\Gamma=\bigcup_{n \in \mathbb{N}} \Gamma_{n}$ and for each $\varepsilon>0$, for each $\gamma_{0} \in \Gamma$ and for each $x^{*} \in B_{X}$. there is $n \in \mathbb{N}$ so that $\gamma_{0} \in \Gamma_{n}$ and $\#\left\{\gamma \in \Gamma_{n} ;\left|\left\langle\gamma, x^{*}\right\rangle\right| \geqslant \varepsilon\right\}<\aleph_{0}$.

The following theorem is a consequence of the previous proposition and the method of proof of [7, Theorem 3].

Proposition 2. Let $X$ be a weakly countably determinedBanach space. Then $X$ contains a bounded total weakly $\sigma$-shrinking subset. Moreover, every total and bounded subset $\Gamma$ of $X$ which countably supports $X^{*}$ is weakly $\sigma$-shrinking.

We can now characterise Gul'ko compacta in $\Sigma(S)$. Our approach is similar to that in [6] and uses ideas in [13].

THEOREM 3. Given a compact subset $K$ of the topological space $\Sigma(S)$, the two following properties are equivalent:

1: $K$ is a Gul'ko compact.

2. There exists a sequence $\left(S_{n}\right)_{n=1}^{\infty}$ of subsets of $S$ such that $S=\bigcup_{n=1}^{\infty} S_{n}$ and given $k \in K, \varepsilon>0$ and $s_{0} \in S$ there exists $n \in \mathbb{N}$ such that $s_{0} \in S_{n}$ and $\#\left\{s \in S_{n}:|k(s)|>\varepsilon\right\}<\aleph_{0}$.

Proof: Given a compact set $K$ in $\Sigma(S)$, let $\pi: S \rightarrow C(K)$ be defined by $\pi(s)(k)$ $:=k(s)$, for all $s \in S, \cdot k \in K$. From the fact that $K$ is in $\Sigma(S)$ it follows that $\# \pi^{-1}(f)$ $\leqslant \aleph_{0}$ for all $f \in \pi(S)$, say $\pi^{-1}(f):=\left\{s_{(f, 1)}, s_{(f, 2)}, \ldots\right\}$ (repeating elements if $\pi^{-1}(f)$ is a finite set). Given $m \in \mathbb{N}$, let $w_{m}: \pi(S) \rightarrow S$ be the selector for the multivalued mapping $\pi^{-1}: \pi(S) \rightarrow 2^{S}$ given by $w_{m}(f):=s_{(f, m)}, f \in \pi(S)$ and let $S_{m}:=w_{m}(\pi(S))$. Obviously, $S=\bigcup_{m \in \mathbb{N}} S_{m}$. Now the mapping $R_{m}:[-1,1]^{S} \rightarrow[-1,1]^{S_{m}}$ defined by $R_{m}(x)(s):=x(s)$ for all $s \in S_{m}$ and $x \in[-1,1]^{S}$, is continuous and $\left.R_{m}\right|_{K}$ is one to one. Thus $K_{m}:=R_{m}(K)$ is homeomorphic to $K$. Let $\pi_{m}: S_{m} \rightarrow C\left(K_{m}\right)$ be defined by $\pi_{m}(s)\left(R_{m}(k)\right):=k(s)$, for all $s \in S_{m}, k \in K . \pi_{m}$ is a one-to-one mapping.

$(1 \Rightarrow 2)$ Fix $m \in \mathbb{N}$. Let $X_{m}:=\overline{\operatorname{span}}\left\{\pi_{m}(s): s \in S_{m}\right\} \subset C\left(K_{m}\right)$. As it was mentioned before, $\left(C\left(K_{m}\right),\|\cdot\|\right)$ is weakly countably determined, and then $\left(B_{\left.C\left(K_{m}\right)^{*}, w^{*}\right) \text { is }}\right.$ angelic (a compact space is angelic if the closure of every subset is attained by sequences), see for example [4, Exercise 12.55]. The set $\pi_{m}\left(S_{m}\right)$ countably supports $K_{m}$ as $K_{m}$ is in $\Sigma\left(S_{m}\right)$. Thus $\pi_{m}\left(S_{m}\right)$ countably supports $\operatorname{conv}\left( \pm K_{m}\right)$ in the dual space and hence 


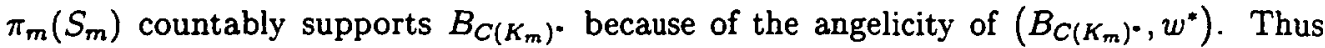
$\pi_{m}\left(S_{m}\right)$ countably supports $B_{X_{\dot{m}}} . X_{m}$ is also weakly countably determined. Apply now Proposition 2 to get $S_{m}=\bigcup_{n=1}^{\infty} S_{m, n}$ from the weakly $\sigma$-shrinking property of $\pi_{m}\left(S_{m}\right)$. Then the family $\left\{S_{m, n}: m, n \in \mathbb{N}\right\}$ gives the conclusion.

$(2 \Rightarrow 1)$ By the definition, we need to prove that $(C(K), p)$ is $\mathcal{K}-c d$. Note that $\pi(S)$ separates points of $K$. From 2 we get a sequence $\left(S_{n}\right)_{n=1}^{\infty}$ of subsets of $S$ such that $\forall k \in K, s \in S, \varepsilon>0$, there exists $n \in \mathbb{N}$ such that $s \in S_{n}$ and

$$
\#\left\{s^{\prime} \in S_{n}:\left|k\left(s^{\prime}\right)\right| \geqslant \varepsilon\right\}<\aleph_{0} .
$$

It follows that $(\pi(S), p)$ is $\mathcal{K}$-cd: indeed, consider the closure $\overline{\pi(S)}$ of $\pi(S)$ in $\left([0,1]^{K}, p\right)$, a compact space. By adding one coordinate if necessary, we can always assume that there exists $s_{0} \in S$ such that $k\left(s_{0}\right)=0, \forall k \in K$. Then $0 \in \pi(S)$. Given $v \in \overline{\pi(S)} \backslash \pi(S)$, there exists $k \in K$ such that $v(k) \neq 0$. Choose $\varepsilon>0$ such that $0<\varepsilon<|v(k)|$. Then, given $s \in S$, we can find, for these $s, k, \varepsilon$, some $n \in \mathbb{N}$ such that (1) holds. It is then obvious that $v \notin \overline{\pi\left(S_{N}\right)}$ and this proves that $(\pi(S), p)$ is $\mathcal{K}$-cd.

Let $W$ be the algebra generated by $\pi(S)$. By the Stone-Weierstrass Theorem it is $\|\cdot\|$-dense, and by elementary properties of $\mathcal{K}-c d$, it is again $\mathcal{K}-c d$. Define the mapping

$$
\Phi:(W, p) \times\left(B_{W}, p\right)^{\mathbf{N}} \mapsto(C(K), p)
$$

given by

$$
\Phi\left(f,\left(f_{n}\right)\right):=f+\sum_{n=1}^{\infty} 2^{-n} f_{n} .
$$

This is a continuous mapping from the $\mathcal{K}$-cd topological space $(W, p) \times\left(B_{W}, p\right)^{\mathbb{N}}$ onto $(C(K), p)$, hence the latter space is also $\mathcal{K}$-cd.

REMARK. A similar proof gives Farmaki's characterisation [8] of Eberlein compacta in $\Sigma(S)$ (see [6]).

We shall now define a property of a Banach space that is related to Gruenhage compacta. Note that in this definition we do not explicitly assume that the unit ball of the dual space is a Corson compact.

Definition 4: We shall say that a Banach space $X$ has property $G$ if $X$ contains a bounded total set $\Gamma$ so that $\Gamma$ can be split into $\Gamma=\bigcup_{n=1}^{\infty} \Gamma_{n}$ in such a way that given $f: g \in B_{X} \cdot, f \neq g$, there are $\gamma \in \Gamma$ and $n \in \mathbb{N}$ such that $|(f-g)(\gamma)|>0, \gamma \in \Gamma_{n}$ and either $\#\left\{\gamma \in \Gamma_{n} ;|f(\gamma)|>|(f-g)(\gamma)| / 4\right\}<\infty$ or $\#\left\{\gamma \in \Gamma_{n} ;|g(\gamma)|>|(f-g)(\gamma)| / 4\right\}<\infty$. REMARK. Clearly; every weakly $\sigma$-shrinking total bounded set in a Banach space $X$ makes $X$ have property $G$. Thus it follows from Proposition 2 that Vasák spaces have property $G$. We conjecture that the non Vašák space $C(\Omega)$ constructed in [1, p. 421] (see also [3. Section 7.3]) has property $G$. 
THEOREM 5. Assume that a Banach space $X$ has property $G$. Then there is an equivalent norm on $X$ the dual of which is strictly convex.

PROOF: Let $\left\{\Gamma_{n}\right\}$ be the collection of all $\Gamma_{n}^{\prime} s$ as in Definition 4. For $n \in \mathbb{N}$, let $\|\cdot\|_{n}$ be a seminorm on $X^{*}$ defined for $f \in X^{*}$ by $\|f\|_{n}:=\left\|(\operatorname{Re})_{n} f\right\|_{D}$, where $(\mathrm{Re})_{n}$ is the operator of the restriction to $\Gamma_{n}$ and $\|\cdot\|_{D}$ is the Day norm on $\ell_{\infty}\left(\Gamma_{n}\right)$. Let the dual equivalent norm $\|\cdot\|$ on $X^{*}$ be defined for $f \in X^{*}$ by

$$
\|f\|^{2}:=\sum_{n=0}^{\infty} \frac{1}{2^{n}}\|f\|_{n}^{2}
$$

where $\|\cdot\|_{0}$ is the original dual norm on $X^{*}$. We shall show that $\|\cdot\|$ is a strictly convex norm on $X^{*}$. Let $f, g \in X^{*}$ be such that $2\|f\|^{2}+2\|g\|^{2}-\|f+g\|^{2}=0$. Then a similar equality holds for each $n$. We may and do assume that $f, g \in B_{X^{*}}$. If $f \neq g$, choose $\gamma \in \Gamma$ so that $\varepsilon:=|(f-g)(\gamma)|>0$ and then choose $\Gamma_{n}$ so that $\gamma \in \Gamma_{n}$ and either $\#\left\{\gamma^{\prime} \in \Gamma_{n} ;\left|f\left(\gamma^{\prime}\right)\right|>\varepsilon / 4\right\}<\aleph_{0}$ or $\#\left\{\gamma^{\prime} \in \Gamma_{n} ;\left|g\left(\gamma^{\prime}\right)\right|>\varepsilon / 4\right\}<\aleph_{0}$. Then by [6, Proposition 4], $\sup _{\gamma^{\prime} \in \Gamma_{n}}\left|(f-g)\left(\gamma^{\prime}\right)\right| \leqslant 3 \varepsilon / 4$, which is a contradiction with $|(f-g)(\gamma)|=\varepsilon$ and $\gamma \in \Gamma_{n}$.

Theorem 5 reminds of a result in [14] which says that a very smooth Banach space $X$ has a dual $X^{*}$ with an equivalent (not necessarily dual) strictly convex norm. However, the space $C\left[0, \omega_{1}\right]$ has an equivalent Fréchet smooth norm and yet does not have property $\mathrm{G}$, since does not have norm whose dual is strictly convex (see, for example, [2]).

From the remark before Theorem 5 we obtain the following

COROLLARY 6. [10] Every Vašák space has an equivalent norm the dual of which is strictly convex.

\section{REFERENCES}

[1] S. Argyros and S. Mercourakis, 'On weakly Lindelöf Banach spaces', Rocky Mountain J. Math. 23 (1993), 395-446.

[2] R. Deville, G. Godefroy and V. Zizler, Smoothness and renormings in Banach spaces, Pitman Monographs 64 (Longman Scientific and Technical, Harlow, 1993).

[3] M. Fabian, Gâteaux differentiability of convex functions and topology. Weak Asplund Spaces (John Wiley \& Sons, New York, 1997).

[4] M. Fabian, P. Habala, P. Hájek, J. Pelant, V. Montesinos and V. Zizler, Functional analysis and infinite dimensional geometry, CMS Books in Mathematics 8 (Springer-Verlag, New York, 2001).

[5] M. Fabian, G. Godefroy, P. Hájek and V. Zizler, 'Hilbert-generated spaces', J. Functional Analysis 200 (2003), 301-323.

[6] M. Fabian, G. Godefroy, V. Montesinos and V. Zizler, 'WCG spaces and their relatives' (to appear).

[7] M. Fabian, V. Montesinos and V.Zizler, 'Biorthogonal systems in weakly Lindelöf spaces', Canad. Math. Bull. (to appear). 
[8] V. Farmaki, 'The structure of Eberlein, uniformly Eberlein and Talagrand compact spaces in $\Sigma\left(\mathbb{R}^{\Gamma}\right)$ ', Fund. Math. 128 (1987), 15-28.

[9] G. Gruenhage, 'A note on Gul'ko compact spaces', Proc. Amer. Math. Soc. 100 (1987), 371-376.

[10] S. Mercourakis, 'On weakly countably determined Banach spaces', Trans. Amer. Math. Soc. 300 (1987), 307-327.

[11] M. Raja, 'Weak* locally uniformly rotund norms and desriptive compact spaces', $J$. Functional Anal. 197 (2003), 1-13.

[12] N.K. Ribarska, 'Internal characterization of fragmentable spaces', Mathematica 34 (1987), 243-257.

[13] G.A. Sokolov, 'On some classes of compact spaces lying in $\Sigma$-products', Comment Math. Univ. Carolin. 25 (1984), 219-231.

[14] D.G. Tacon, 'The conjugate of a smooth Banach space', Bull. Austral. Math. Soc. 2 (1970), 415-425.

Mathematical Institute of the Czech Academy of Sciences

Žitná 25, 11567

Prague 1

Czech Republic

e-mail: fabian@math.cas.cz
Departamento de Matemática Aplicada

E.T.S.I. Telecomunicación

Universidad Politécnica de Valencia

C/Vera, s/n. 46071 Valencia

Spain

e-mail: vmontesinos@mat.upv.es

Department of Mathematical Sciences

University of Alberta

632 Central Academic Building

Edmonton, Alberta T6G 2G1

Canada

e-mail: vzizler@math.ualberta.ca 